\title{
CONTROLADOR DE VELOCIDADE APLICADO A UMA TURBINA HIDRÁULICA DE FLUXO CRUZADO'
}

\author{
Anderson Lopes Jacondino², Murilo Vargas da Cunha ${ }^{3}$, Cristian Rogério Guidotti Aguiar² \\ 1 Aceito para publicação no $3^{\circ}$ trimestre de 2013. \\ 2 Programa de Pós-Graduação e Engenharia Elétrica da Universidade Federal de Santa Maria e Universidade \\ Católica de Pelotas \\ ${ }^{3}$ Universidade Católica de Pelotas e Docente do Instituto Federal de Educação, Ciência e Tecnologia Sul-Rio- \\ Grandense
}

Palavras-chave: sistema automatizado, controle de velocidade, sistema isolado.

\section{Resumo}

O trabalho apresenta o desenvolvimento e montagem de um sistema automatizado de controle de velocidade em malha fechada, aplicado a uma turbina hidráulica de fluxo cruzado, tipo Banki, para funcionamento como parte de uma Micro Central Hidrelétrica ( $\mathrm{MCH})$, em pequenos sistemas isolados. Uma vez verificados os parâmetros de operação do sistema partiu-se para o desenvolvimento da lógica do programa do controlador e desenvolvimento do transdutor de velocidade. Com a finalidade de validar o sistema desenvolvido, foi montada uma planta em laboratório, sendo aplicado o protótipo do sistema de controle a uma turbina. O sistema desenvolvido satisfez todos os critérios estabelecidos nos Procedimentos de Distribuição de Energia Elétrica no Sistema Elétrico Nacional (PRODIST), Módulo 8, da Agência Nacional de Energia Elétrica.

\begin{abstract}
The paper shows the development and the installation of an automated system of speed control in a closed loop, applied to a cross-flow hydraulic turbine, Banki type, for operation as part of a Micro Hydroelectric Power Plant, in small isolated systems. Once, it was verified the operating parameters of the system, the logical development of the controller program and the
\end{abstract}


development of the speed transducer were worked on. In order to validate the developed system, it was assembled a plant in a laboratory and applied the prototype of the control system to a turbine. The developed system met all the established criteria in the Distribution Procedures of Electrical Energy in the National Electric System (PRODIST), Module 8, from the National Agency of Electricity.

Keywords: automated system, speed control, isolated systems.

\section{Introdução}

Em um sistema isolado, o conjunto turbogerador necessita, para o seu adequado funcionamento de controladores para que mantenham os valores de frequência e tensão dentro dos parâmetros desejados.

As variações de carga no gerador podem gerar distúrbios na frequência (velocidade de rotação da turbina) e na tensão de saída do gerador. (Vanfretti, 2005). As variações de potência reativa afetam basicamente a amplitude da tensão enquanto que a variação da potência ativa afeta basicamente a frequência do sistema. Portanto, é possível controlar o fornecimento de potência ativa de forma independente da reativa (Vanfretti, 2007). O sistema de controle de potência ativa monitora a frequência (velocidade de rotação do gerador) e atua de maneira a variar o torque no eixo da máquina primária (turbina).

A função básica do sistema de controle em malha fechada (SCMF) de potência ativa é corrigir a frequência do sistema na ocorrência de um distúrbio, comparando o sinal de erro com um valor de referência, realimentando o controlador de maneira a acertar a saída do sistema ao valor desejado (Pallás-Areny, 1991).

Conforme Negri (2002), o controle em pequenas e micro centrais hidrelétricas em sistemas isolados pode ser feito por Controlador Lógico Programável (CLP), através da leitura do processo por sensores e transdutores e através de uma lógica programada o processo é corrigido através de atuadores.

Neste trabalho é apresentada a concepção de um sistema de controle de velocidade de uma turbina hidráulica do tipo Banki para micro central hidrelétrica e validada em planta montada em laboratório.

\section{Materiais e métodos}

Revista Brasileira de Energias Renováveis, v. 2, p. 36-48, 2013 
O diagrama do sistema de controle proposto, conforme Figura 1, foi projetado para corrigir a velocidade de rotação do eixo da turbina na ocorrência de distúrbios, de forma a não permitir que os valores de frequência ultrapassem os limites inferior e superior, e também, não permitindo que o tempo de correção ultrapasse o valor correspondente à faixa de operação de frequência, sendo ambos valores, estabelecidos pelos Procedimentos de Distribuição de Energia Elétrica no Sistema Elétrico Nacional, Módulo 8 (ANEEL, 2011). Caso os parâmetros não sejam obedecidos, a carga é desconectada dos terminais do gerador. O Sistema desenvolvido pode ser descrito da seguinte maneira: através de um transdutor, que consiste em um disco acoplado ao eixo da turbina, com 30 ranhuras capazes de interromper o sinal de um fotoacoplador, é gerado um trem de pulsos com frequência diretamente proporcional ao número de ranhuras do disco e da velocidade angular do eixo da turbina. Um conversor de frequência/ tensão, exibido na Figura 2, converte o trem de pulsos em um nível de tensão diretamente proporcional a frequência de entrada, ou seja, da velocidade do eixo da turbina (Turchetti, 2007).

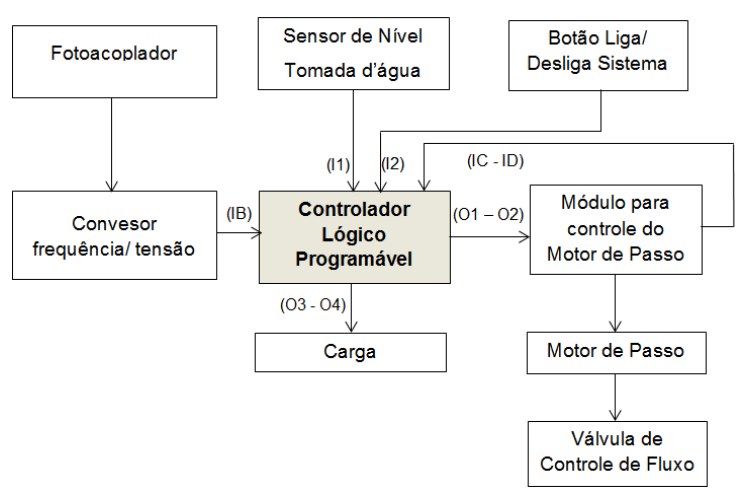

Figura 1. Diagrama do Sistema de Controle de Velocidade

Como se pode verificar nas Figuras 1 e 3, os indicadores I1 e I2 correspondem a entradas digitais do Controlador Lógico Programável, como IB, IC e ID correspondem a entradas analógicas, e também, O1, O2, O3 e O4 correspondem a saídas digitais. 


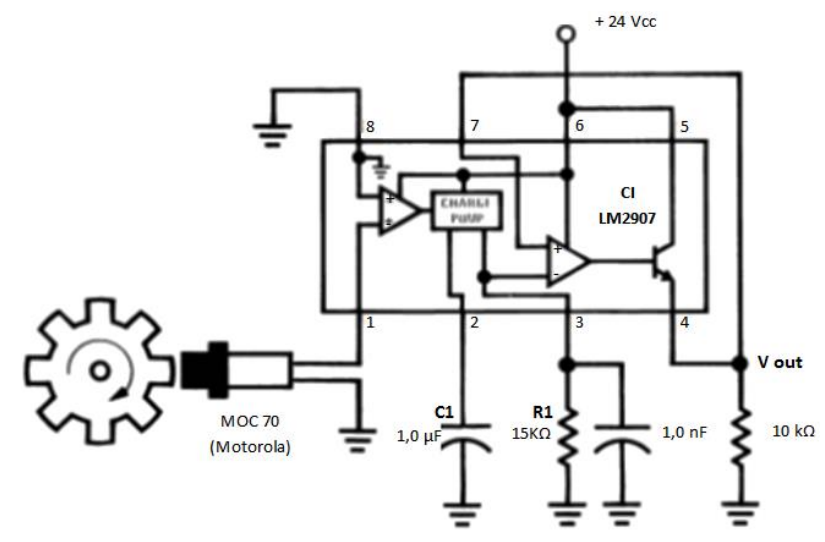

Figura 2. Esquema básico de ligação do Conversor Frequência/Tensão

A relação de frequência de entrada por tensão de saída $\left(\mathrm{V}_{\text {out }}\right)$ do conversor frequência/ tensão pode ser calculada pela multiplicação do valor de frequência de entrada por um Volt de saída ( $\left.\mathrm{f}_{\mathrm{IN}}\right)$, pela tensão de alimentação do circuito $\left(\mathrm{V}_{\mathrm{cc}}\right)$, pelo valor da resistência,

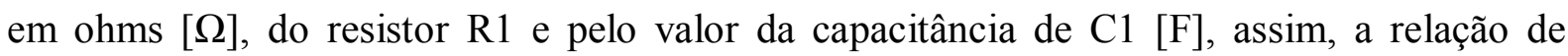
frequência/ tensão pode ser calculada pela Equação 1 (National, 2009).

$$
V_{\text {out }}=f_{I N} \cdot V_{c c} \cdot R_{1} \cdot C_{1}
$$

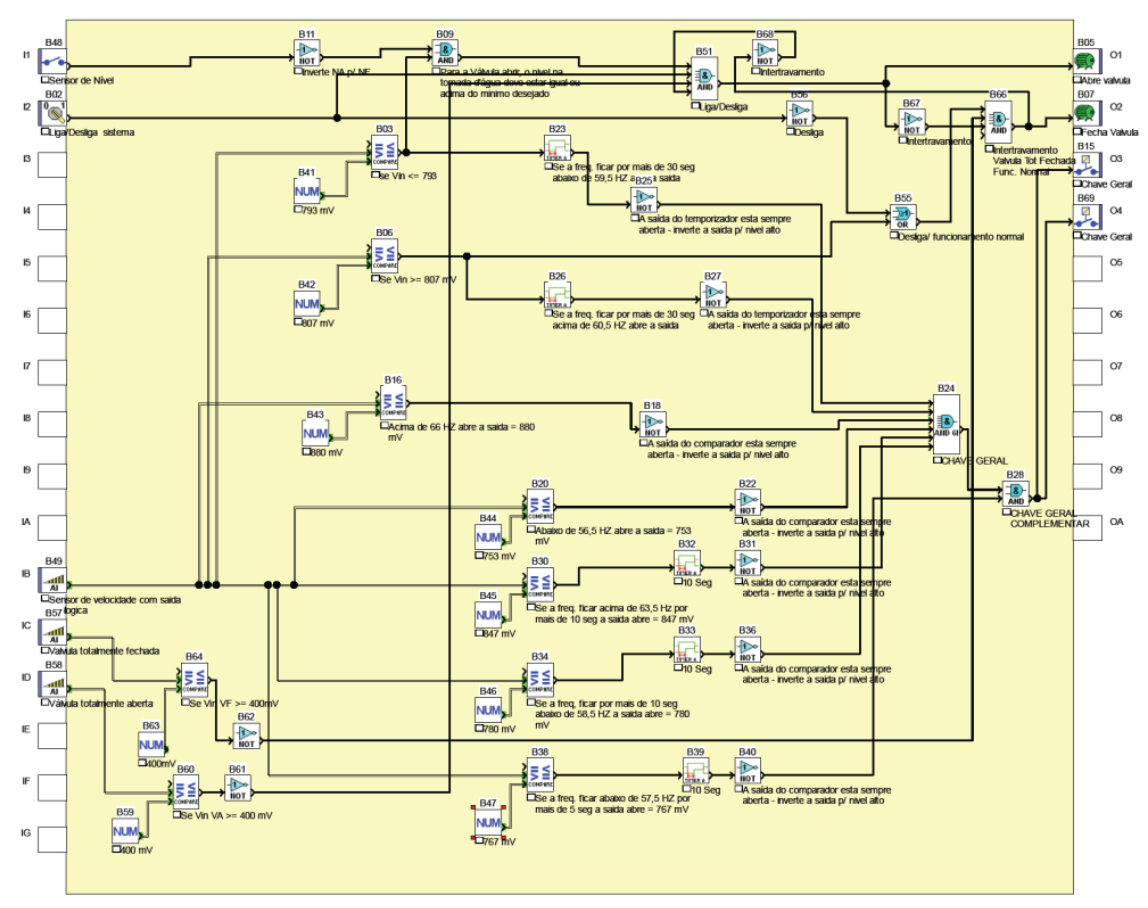

Figura 3. Lógica do Programa - CLP 
O Controlador Lógico Programável da marca Crouzet, modelo XD26, de fabricação italiana, monitora o estado do processo através do nível de tensão do conversor e de acordo com a lógica estabelecida, conforme exibido na Figura 3, atua no processo variando o fluxo d'água que passa pela turbina. A lógica foi construída através da Linguagem gráfica de programação FBD (Function Block Diagram), no software de programação do controlador lógico programável, tendo como parâmetros os valores de frequência e os períodos de operação nos respectivos níveis de frequência descritos nos Procedimentos de Distribuição de Energia Elétrica no Sistema Elétrico Nacional (ANEEL, 2011).

O conjunto atuador, exibido na Figura 4, é constituído por uma válvula do tipo gaveta de $50[\mathrm{~mm}]$ de diâmetro, acoplada diretamente ao eixo de um motor de passo híbrido, unipolar, da fabricante Minebea, com torque de 32 [kgf.cm] juntamente com seu o módulo de controle.

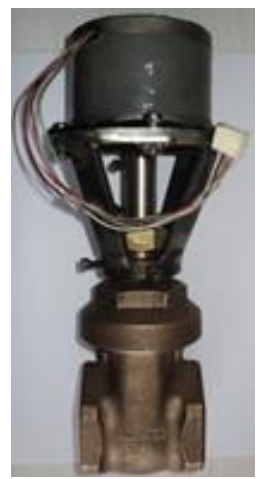

Figura 4. Conjunto atuador

\section{Funcionamento do Sistema de Controle}

O sistema de controle foi desenvolvido para atuar diretamente sobre a vazão de água que passa pela turbina hidráulica, assim, consequentemente, agindo sobre a velocidade angular do eixo da turbina, que corresponde diretamente à frequência e tensão nos terminais do gerador. Considerando que esta turbina foi projetada para ser acoplada através de um conjunto de polias, com uma relação de 1:4, em um gerador elétrico de 4 polos, 1800 [rpm], deve-se manter a velocidade do eixo da turbina entre 446,25 e 453,75 [rpm], ou seja, entre 59,5 e 50,5 [Hz]. A velocidade do eixo da turbina é monitorada pelo Controlador Lógico Programável através do nível de tensão $\mathrm{V}_{\text {out }}$ nos terminais do conversor frequência/ tensão, e atua sobre o processo com os requisitos pré-estabelecidos, que são os exibidos na Tabela 1. 
Como se pode notar na Tabela 1, para cada faixa de operação de frequência exibido na Tabela 2, há uma faixa de tensão $\mathrm{V}_{\text {out }}$ correspondente nos terminais do conversor frequência/ tensão.

Tabela 1. Parâmetros do sistema de controle.

\begin{tabular}{l|l|l}
\hline \hline $\begin{array}{c}\text { Freq. } \\
{[\mathbf{H z}]}\end{array}$ & $\begin{array}{c}\text { Tensão Vout } \\
\text { Conversor } \\
{[\mathbf{V}]}\end{array}$ & $\begin{array}{c}\text { Velocidade } \\
\text { do eixo da } \\
\text { turbina } \\
{[\mathbf{r p m}]}\end{array}$ \\
\hline 56,5 & 7,53 & 423,75 \\
\hline 57,5 & 7,67 & 431,25 \\
\hline 58,5 & 7,80 & 438,75 \\
\hline 59,5 & 7,93 & 446,25 \\
\hline 60,0 & 8,00 & 450,00 \\
\hline 60,5 & 8,07 & 453,75 \\
\hline 63,5 & 8,47 & 476,25 \\
\hline 66,0 & 8,80 & 495,00 \\
\hline \hline
\end{tabular}

Como se pode notar na Tabela 2, para o nível de frequência, que esteja fora da faixa de operação estabelecida pelo PRODIST - Módulo 8, ou seja, entre 59,5 [Hz] e 60,5 [Hz], demanda-se a atuação do sistema de controle, abrindo ou fechando a válvula do conjunto atuador para respectivamente aumentar ou diminuir a vazão d'água e consequentemente variar a velocidade do eixo do gerador (ANEEL, 2011).

Tabela 2. Ações do sistema de controle.

\begin{tabular}{l|l|l}
\hline \hline \multirow{2}{*}{ Freq. [Hz] } & \multicolumn{2}{|c}{ Ação desejada } \\
\cline { 2 - 3 } & $\begin{array}{c}\text { Válvula } \\
\text { de } \\
\text { Controle }\end{array}$ & $\begin{array}{c}\text { Contatos de saída } \\
\text { (Gerador) }\end{array}$ \\
\hline $\mathrm{f}<56,5$ & Abre & Abrem \\
\hline $56,5 \leq \mathrm{f}<57,5$ & Abre & Abrem após 5 seg. \\
\hline $57,5 \leq \mathrm{f}<58,5$ & Abre & Abrem após 10seg. \\
\hline
\end{tabular}




\begin{tabular}{l|l|l}
\hline $58,5 \leq \mathrm{f}<59,5$ & Abre & Abrem após 30seg. \\
\hline $59,5 \leq \mathrm{f} \leq 60,5$ & $\begin{array}{l}\text { Mantém } \\
\text { aberta }\end{array}$ & Mantém fechado \\
\hline $60,5<\mathrm{f} \leq 63,5$ & Fecha & Abrem após 30seg. \\
\hline $63,5<\mathrm{f} \leq 66,0$ & Fecha & Abrem após 10seg. \\
\hline $66,0<\mathrm{f}$ & Fecha & Abrem \\
\hline \hline
\end{tabular}

Os períodos de tempo estabelecidos para correção do nível de frequência na Tabela 2, correspondem ao tempo máximo de operação do sistema com nível de frequência fora da faixa de operação também estabelecidos no PRODIST - Módulo 8. Nota-se também, que a carga é desconectada dos terminais do gerador caso o nível de frequência fique fora da faixa de operação por tempo superior ao estipulado.

\section{Elementos da planta montada}

\section{Turbina}

Nos testes em laboratório foi utilizada uma turbina de fluxo cruzado, também conhecida como Turbina Banki, de velocidade de operação de 450 [rpm], para uma tubulação forçada de 50 [mm] de diâmetro, desenhada para uma altura de queda d'água de 20 [m] e com potencia no eixo de $0,2[\mathrm{~kW}]$.

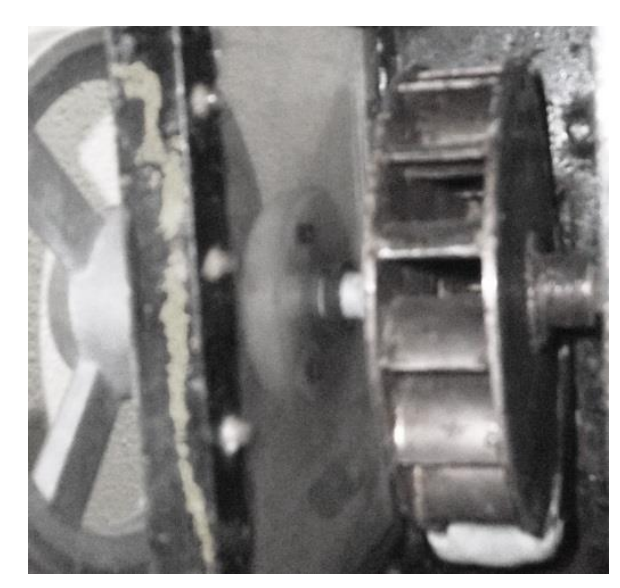

Figura 5. Turbina Banki 


\section{Planta montada}

$\mathrm{Na}$ Figura 6, é exibido o esquema da planta montada para experimentação em laboratório; deve-se ressaltar que em todas as provas a carga aplicada aos terminais do gerador é simulada por um sistema de freio, conhecido como Freio de Prony; isto foi feito visto que o sistema de controle de velocidade da turbina é independente, ou seja, o controle de tensão e velocidade pode ser feito de forma independente.

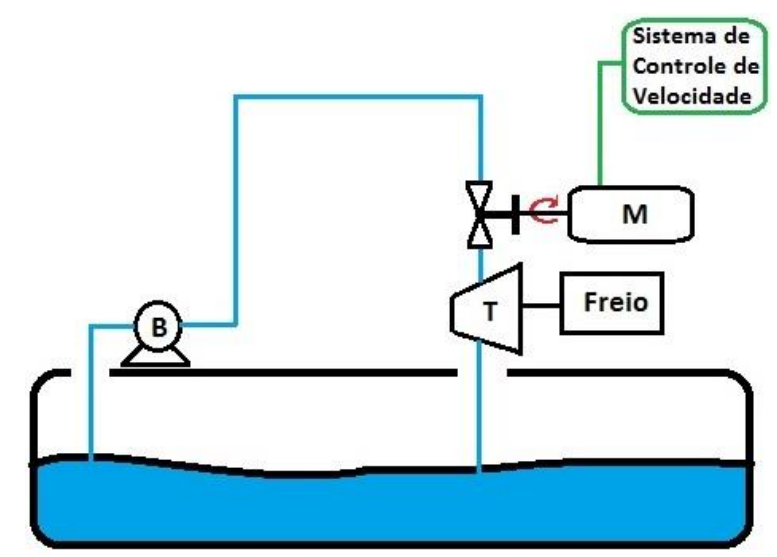

Figura 6. Esquema da planta montada

Os parâmetros a serem estimados nos ensaios são os seguintes: tempo de resposta do sistema e o percentual da carga máxima que o sistema suporta sem que a velocidade (frequência) fique abaixo dos níveis estabelecidos pela ANEEL (2011).

\section{Resultados e discussão}

A validação do sistema de controle aplicado a uma turina Banki foi realizada em duas etapas; na primeira etapa foi aferido o conversor frequência/ tensão com o auxílio de um gerador de funções, um multímetro e um osciloscópio; o disco com 30 ranhuras foi acoplado ao eixo de um motor de velocidade controlada, logo após foi instalado o fotoacoplador e colocado o motor em funcionamento, variando a velocidade de 0 à 2000 [rpm] com espaçamentos de 100 [rpm], desta forma, foi verificada a amplitude, forma de onda e frequência do sinal gerado. A forma de onda verificada, que foi uma onda quadrada com amplitude de 3,81 [V], foi reproduzida em seus diversos níveis de frequência pelo gerador de funções e aplicada no conversor frequência/ tensão, assim, com o auxilio dos equipamentos de medição e através do potenciômetro instalado como resistor R1, conforme expõe a Figura 2, foi calibrada a saída de tensão $\left(\mathrm{V}_{\text {out }}\right)$, sendo gerado o gráfico exibido na Figura 7. 


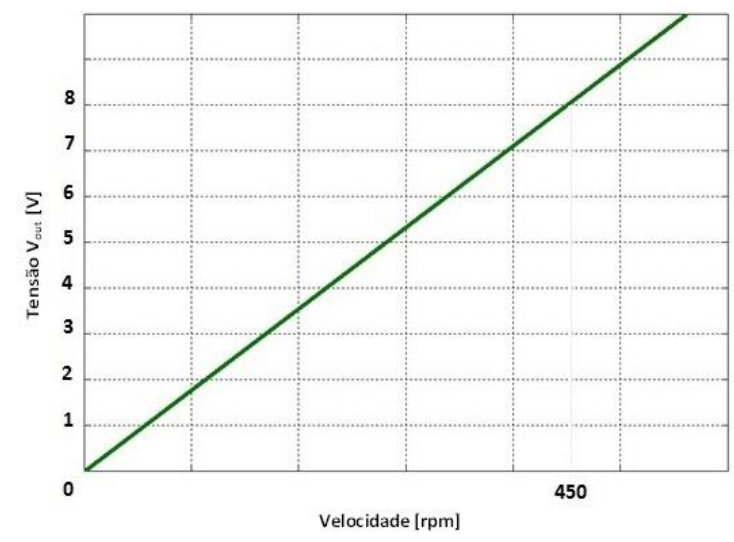

Figura 7. Gráfico de relação entre Tensão $\mathrm{V}_{\text {out }}$ e velocidade do eixo da turbina

A segunda etapa consistiu em validar o sistema de controle de forma integral, verificando o valor de carga máxima suportada pela turbina, comportamento do sistema em resposta a distúrbios e o percentual de carga suportada pela turbina sem que o sistema ultrapasse os níveis de frequência estipulado pelo PRODIST - Módulo 8. Para tanto, foi montado o esquema da planta exibido na Figura 6. O valor de carga máxima suportada pela turbina foi obtido da seguinte forma: a planta montada foi reconfigurada de forma que o sistema de controle foi suprimido, ou seja, a válvula de controle foi mantida totalmente aberta; em seguida o sistema foi colocado em funcionamento, e após a velocidade do eixo da turbina entrar em regime permanente, ou seja, atingindo a velocidade máxima sem aplicação de carga, foi aplicado o freio ao eixo da turbina, conhecido como Freio de Prony, e foi verificado a força de 560 [gf] para reduzir a velocidade ao valor mínimo aceitável, correspondente à 60 [Hz], que é de 450 [rpm] conforme é exposto na Tabela 1.

Para análise do comportamento do sistema em resposta a distúrbios e verificação do percentual de carga suportada pela turbina sem que o sistema ultrapasse os níveis de frequência estipulado pelo PRODIST, a planta foi montada de forma integral. Após aplicado vários percentuais da carga máxima suportada pela turbina através do freio foi verificado que o sistema exibido na Figura 3 atuou de forma a suprir todos os parâmetros estabelecidos; e aplicando 9\% de carga houve a redução de velocidade da máquina primária ao limite máximo inferior, de 423,75 [rpm] representando 56,5 [Hz]. O tempo necessário para que o sistema corrigisse a frequência ao nível almejado, contido entre 59,5 e 60,5 [Hz] ou velocidade contido entre 446,25 e 453,75 [rpm] é inferior a 10 segundos [s], como pode-se verificar na Figura 8. 


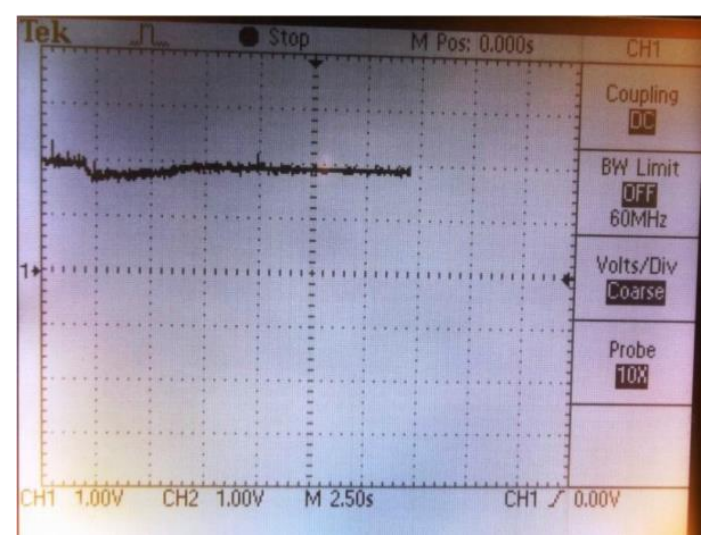

Figura 8. Resposta do sistema de controle de velocidade com $9 \%$ de carga

\section{Conclusões}

O trabalho apresentado possui aplicação direta em sistemas de controle de velocidade em Micro Centrais Hidrelétricas, mais especificamente, em turbinas de fluxo cruzado, também conhecidas como turbinas do tipo Banki. A seguir são apresentadas as principais conclusões do sistema. O conversor de frequência/ tensão com o CI LM2907 se mostrou uma boa alternativa como transdutor, pois apresenta uma tensão de saída diretamente proporcional à frequência de entrada. Durante os ensaios foi possível comprovar a eficácia do sistema de controle desenvolvido através do gráfico de resposta do sistema a uma perturbação. Com a planta montada foi possível também, verificar o percentual aceitável de carga aplicada em um único passo, que foi de $9 \%$ com intervalo mínimo de tempo entre a inserção de novas cargas de 10 [s]. Período este, que corresponde ao tempo de recuperação do sistema, pois devido a atuação do sistema de controle, o nível de frequência é elevado até a faixa contida entre 57,5 e $58,5[\mathrm{~Hz}]$ num período inferior a 5 [s] e em ação contínua do sistema, o nível de frequência é elevado até a faixa contida entre 56,5 e $57,5[\mathrm{~Hz}]$ em período inferior a 5 [s], totalizando assim, um período máximo de 10 [s] para aplicação de novas cargas. O sistema desenvolvido em sua forma integral satisfez as necessidades relativas à qualidade da energia elétrica, abordando a qualidade do produto, e também, possibilitou a verificação do limite de adição de carga aplicado à Micro Central Hidrelétrica, num único passo, observando o tempo de resposta do sistema a distúrbios.

\section{Agradecimentos}

Os autores agradecem aos laboratoristas e pesquisadores do Laboratório de Energias Renováveis da Universidade Luterana do Brasil - Campus Canoas/ RS, ao Programa de Pós- 
JACONDINO A. L., et al.,

Graduação em Engenharia Elétrica da Universidade Federal de Santa Maria e também, aos Professores curso de Engenharia Elétrica da Universidade Católica de Pelotas/ RS. 


\section{Referências}

ANEEL (2011). PRODIST - Procedimentos de Distribuição de Energia Elétrica no Sistema Elétrico Nacional. Disponível em:

<http://www.aneel.gov.br/arquivos/PDF/Módulo8_Revisão_4.pdf>. Acesso em: 29 abril 2012.

National (2009). Catálogo Semicondutor LM 2907. Disponível em:

<http://datasheetcatalog.com/>. Acesso em: 15 julho 2012.

Negri, V. L. (2004), Capacitação Industrial para Construção de Sistemas Hidráulicos de Controladores de Turbinas e Modelagem para Automação de Pequenas Centrais

Hidrelétricas, UFSC, Brasil.

Pallás-Areny, R.; Webster, J. G. (1991). Sensors and Signal Conditioning. New York: John Wiley \& Sons.

Ribeiro, M. A. (2003), Válvulas de Controle e Segurança. $5^{\text {a }}$ ed. Salvador/ BA.

Turchetti, V. A., (2007), Estudo de Encoder's Ópticos Baseados no Efeito Moiré e Projeto de um Kit de Encoder's Didático, UEC, Brasil.

Vanfretti, L. (2005), Modelación y Simulación de la Máquina Síncrona y su Operación em Sistemas de Potencia, USAC, Guatemala.

Vanfretti, L. (2007), Sistemas de Control de Excitación y Estabilizadores de Sistemas de Potencia RPI, USA. 